\title{
Conectivismo: Hacia El Nuevo Paradigma De La Enseñanza Por Competencias
}

\author{
Marisa Cecilia Tumino \\ Juan Manuel Bournissen \\ Universidad Adventista del Plata
}

doi: 10.19044/esj.2016.v12n10p112 URL:http://dx.doi.org/10.19044/esj.2016.v12n10p112

\begin{abstract}
The three main learning theories most frequently used in the design and creation of instructional environments are behaviorism, cognitivism and constructivism. In the last years, technology has reorganized the way we live, communicate and learn. The arrival of the web 2.0 has greatly increased students' access to the interconnected information required for their professional formation. Scientific and technological progress has impelled innovation as an assertive paradigm of the well-meant development efforts of a knowledge society in continuous transformation. One of the great challenges faced by educational institutions is that of training competent professionals qualified to adapt themselves to the ceaseless changes and challenges posed by each discipline, with the consequent responsibility of implementing teaching and evaluation techniques which would allow to identify the degree of achievement of professional competencies aimed to develop in the students. This essay addresses the way in which Informatics -as a science which studies methods, processes, and storage, processing and transmission techniquescan lay the foundations this changes convey and introduce innovative practices aimed to get hold of the benefits of connectivism to strengthen competencies of the professional profile. According to Bartolomé (2011), in connectivism -as a learning theory first introduced by Siemens and Downeslearning is produced through a process of connecting and generating information in the context of a learning community, where this community acts as a node, part of a bigger net with other nodes sharing resources with each other. The size and strength of the nodes varies according to the concentration of information and the number of individuals participating in the node. Knowledge is distributed throughout the net. Some interesting concepts are the continuous change of validity and precision of information (knowledge dynamics) and also the change in the students' skills over time. Drawing from the information gathered in this study, it is believed that the
\end{abstract}


self-determined learning methodology, along with the resources gained through connectivism, creates a beneficial scenario for the professional training aimed to develop the competences demanded by society.

Keywords: Connectivism, Self-determined learning, Educational technologies, Learning process

\section{Resumen}

El conductismo, el cognitivismo y el constructivismo son las tres grandes teorías de aprendizaje utilizadas más a menudo en la creación de ambientes instruccionales. En los últimos años la tecnología ha reorganizado la forma en la que vivimos, nos comunicamos y aprendemos. La aparición de la web 2.0 ha aumentado considerablemente el acceso de los estudiantes a la información interconectada que demanda su formación profesional. El progreso científico-tecnológico ha impulsado la innovación como paradigma asertivo de los bienintencionados esfuerzos de desarrollo que responden a los cuestionamientos de una sociedad del conocimiento en constante transformación. Uno de los grandes retos de las instituciones educativas consiste en formar profesionales competentes capacitados para adaptarse a los incesantes cambios y desafíos que propone cada disciplina, con la consiguiente responsabilidad de implementar estrategias de enseñanza y evaluación que permitan identificar el grado de logro de las competencias profesionales a las que aspiran desarrollar en los estudiantes. El presente ensayo aborda la manera en que la Informática, como ciencia que estudia los métodos, los procesos y las técnicas de almacenamiento, procesamiento y transmisión de información, puede sentar las bases que acompañan estos cambios e introducir prácticas innovadoras tendientes a apropiarse de las virtudes del conectivismo en función del fortalecimiento de competencias que hacen al perfil profesional. Según Bartolomé (2011), en el Conectivismo, como teoría del aprendizaje inicialmente propuesta por Siemens y Downes, el aprendizaje se produce a través de un proceso de conectar y generar información en el contexto de una comunidad de aprendizaje, donde esta comunidad actúa como un nodo, parte de una red más amplia con otros nodos que comparten entre sí recursos. El tamaño y fuerza de los nodos varían de acuerdo con la concentración de información y el número de individuos participando del nodo. El conocimiento es distribuido en la red. Algunos conceptos interesantes son el continuo cambio de la validez y precisión de la información (dinamismo del conocimiento) e igualmente el cambio en las habilidades del estudiante a lo largo del tiempo. A partir de la información recabada en el estudio, se estima que la metodología de aprendizaje auto-determinado, acompañado por los recursos que proporciona el conectivismo, crea un escenario beneficioso para la 
formación profesional que persigue el desarrollo de las competencias demandadas por la sociedad.

Palabras clave: Conectivismo, Aprendizaje auto-determinado, Tecnologías educativas, Proceso de enseñanza aprendizaje

\section{Introducción}

El conductismo, el cognitivismo y el constructivismo son las tres grandes teorías de aprendizaje utilizadas más a menudo en la creación de ambientes instruccionales. Estas teorías, sin embargo, fueron desarrolladas en una época en la que el aprendizaje no había sido impactado por la tecnología. En los últimos años la tecnología ha reorganizado la forma en la que nos comunicamos y aprendemos.

La idea clave reside en el hecho de que "el aprendizaje es un proceso de formación de redes que tiene como agente principal al propio individuo". $\mathrm{La}$ red conecta nodos que representan entidades externas tales como personas, organizaciones, bibliotecas, sitios web, wikis, libros, revistas y bases de datos, entre otros. "El acto de aprender consiste en crear una red externa donde los nodos se conectan para dar forma a una compleja fuente de conocimiento." 1

Según Laborda (2005), introducir las TIC en la educación implica muchos cambios en el proceso de enseñanza-aprendizaje. Estas modificaciones se ven reflejadas en el tiempo, en el espacio y en los roles asumidos por los diferentes actores, como se muestra en la Figura 1, lo que lleva a rediseñar el contexto educativo.

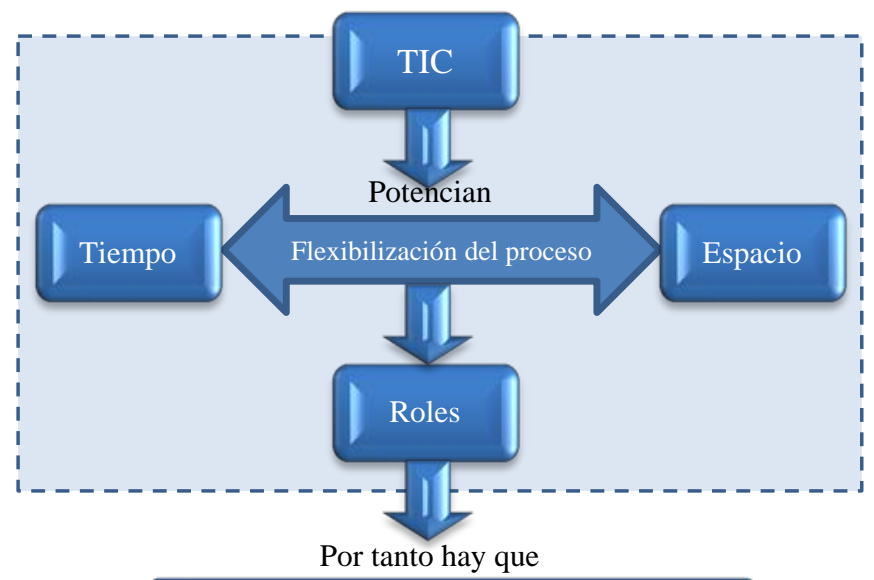

Rediseñar el contexto educativo

Figura 1: Contexto educativo

\footnotetext{
${ }^{1}$ http://canaltic.com/blog/?p=800
} 
Entre los cambios que se producen se pueden mencionar los siguientes:

\section{Cambios en el proceso educativo}

Los constantes cambios en la sociedad del Siglo XXI llevan aparejado nuevas formas de relacionarnos, de comunicarnos y de interactuar. La información y el conocimiento tienen cada vez mayor influencia en el entorno laboral y personal, lo que conlleva cambios en los entornos profesionales que, sumado a una mayor diversificación de la población estudiantil, exige cambios en el sistema educativo, regido por el principio de igualdad de oportunidades y la no discriminación, que dé respuesta a la nueva sociedad de la información.

Para Laborda (2005), se trata, no ya de enseñar sobre TIC, es decir de formar en las habilidades y destrezas que son necesarias para desenvolverse con soltura en la sociedad de la información; sino de dar un paso más y entender que utilizar las TIC en la educación significa seleccionar algunas de las herramientas que ofrecen las TIC y usarlas desde una perspectiva pedagógica, pero no como un complemento de la enseñanza tradicional sino como una vía innovadora que, integrando la tecnología en el currículo, consigue mejorar los procesos de enseñanza-aprendizaje y los progresos académicos de los estudiantes. Se trata, pues, de enseñar con y a través de las TIC, además de sobre TIC o de TIC.

El reconocimiento de que la tecnología está propiciando la relación e interconexión de información, procesos y conocimientos de personas de diversas culturas de la manera más estrecha, inmediata y global. En este sentido, el progreso de la ciencia compromete enfoques metodológicos y referentes dialécticos diferentes que, entre otros aspectos, introducen la conectividad como un nuevo paradigma en los saberes y haceres. El aprendizaje no se vería favorecido si no se aceptase la posibilidad de mejorar las propias acciones mediante la corrección y la aceptación de la realidad compartida por los demás actores escolares. "La información ha cambiado el sentido del saber y hacer hacia el asentamiento de nuevos paradigmas planteados desde la tecnología” (Padrón y Ortega, 2012: 141).

\section{Cambios en el objeto de la enseñanza}

El objeto de la enseñanza es la transmisión de los conocimientos, pero con la llegada de las TIC a la educación, se cambia el foco de la misma para centrarlo en el alumno. Hay que enseñar a aprender para que este aprendizaje le sirva para toda la vida, lo que implica que hay que formar en competencias para que el alumno se adapte a la sociedad en constante cambio. En la actualidad se sebe dejar el aprendizaje memorístico tan utilizado en la educación tradicional previa al Siglo XX. 
El conectivismo promueve que la capacidad de saber más es más crítica que aquello que se sabe en un momento dado. Además sostiene que la habilidad de distinguir entre la información relevante y la irrelevante resulta vital. También es crítica la habilidad de reconocer cuándo una nueva información altera un entorno basado en las decisiones tomadas anteriormente. Por lo tanto nuestra habilidad para aprender lo que necesitamos mañana es más importante que lo que sabemos hoy (Siemens, 2004).

\section{Cambios en los objetos educativos}

Los docentes deben capacitar a sus alumnos para vivir en la sociedad de la información y del conocimiento. Por lo tanto hoy se debe crear conocimiento de valor que perdure en el tiempo, y el alumno debe aprender a manejar la información eficaz y responsablemente, para de esa forma poder solucionar los problemas que se le presentan de la mejor manera.

Marquès Graells (2006) señala que los docentes del siglo XXI deberían estar capacitados para poder utilizar en forma transparente, es decir sin tener que recurrir a ayuda alguna, los siguientes tópicos, tal como se aprecia en la Figura 2:

1. Saber utilizar las principales herramientas de Internet.

2. Conocer las características básicas de los equipos.

3. Diagnosticar qué información se necesita, y saberla encontrarla

4. Evaluar la calidad y la idoneidad de la información obtenida.

5. Saber aprovechar las posibilidades de comunicación en Internet.

6. Conocer las funciones básicas de un paquete de oficina.

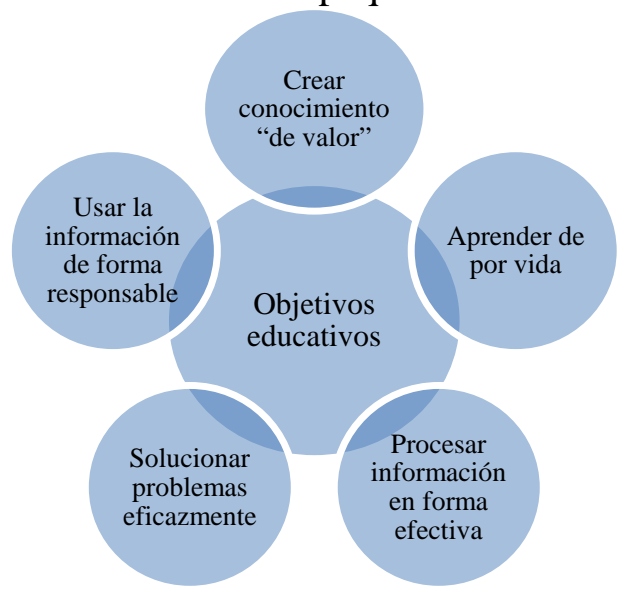

Figura 2: Objetivos educativos

\section{Cambios en los centros educativos}

Para la inclusión de las TIC en los centros educativos se requiere pensar en cambios. Se necesitan equipos en red (con servidores) y una 
conexión a Internet con un ancho de banda adecuado a la necesidad de la institución, teniendo en cuenta la cantidad de usuarios que podrían estar conectados en forma simultánea. Esto conlleva a que la institución debe velar por la capacitación de personal profesional en el área de las TIC y de personal docente que debe usar las TIC en forma transparente. Adicionalmente los centros educativos deben tener una política para integrar las TIC y mantener su actualización, como se refleja por Laborda (2005) en la Figura 3.

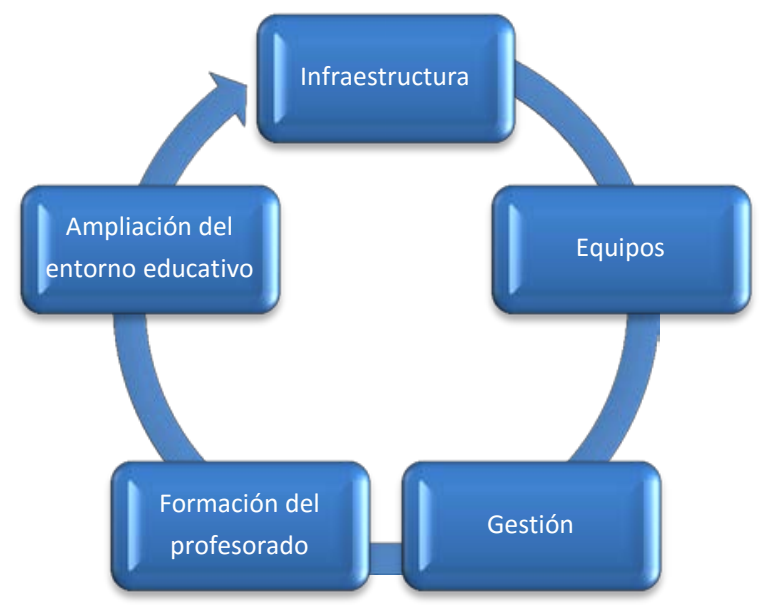

Figura 3, construida a partir de Laborda (2005)

\section{Cambios en las formas pedagógicas}

Los cambios que se producen por la inclusión de las TIC en educación provocan cambios en el rol de los actores intervinientes, como se sintetiza en la Figura 4.

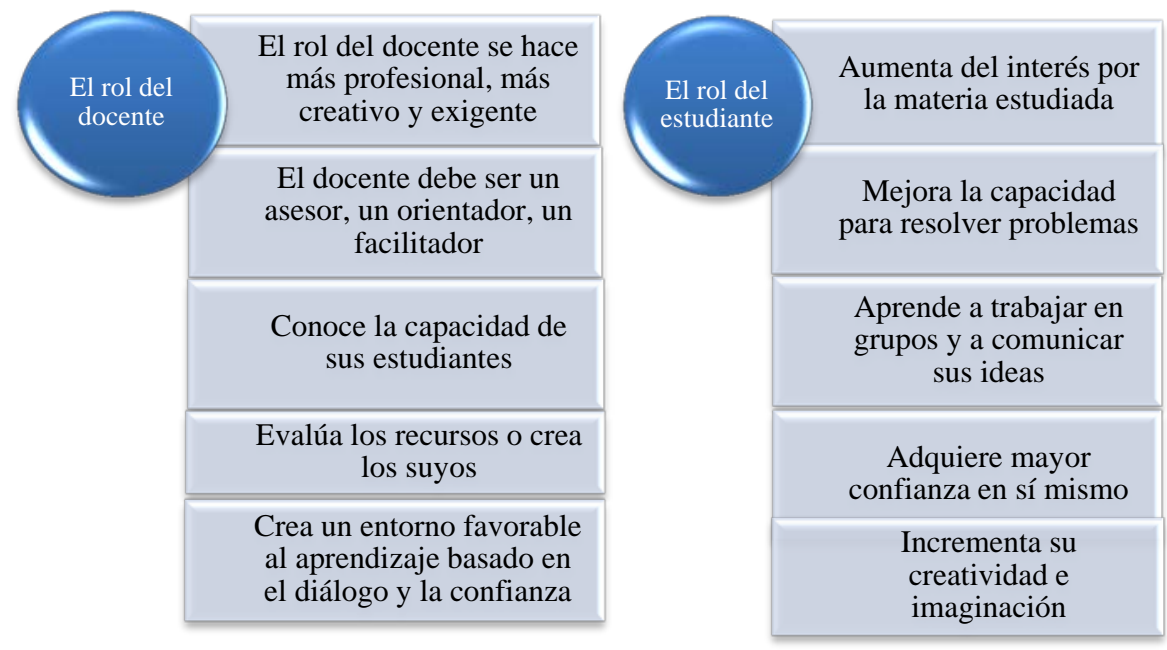

Figura 4, construida a partir de Laborda (2005) 
El uso de Internet en educación proporciona la oportunidad de usar metodologías más activas y motivadoras, que acercan la realidad profesional a la etapa formativa y permiten la adquisición de otras capacidades aparte de las directamente vinculadas al conocimiento científico técnico de la profesión (Barragán Sánchez, Mimbrero Mallado y Pacheco GonzálezPiñal, 2013: 13)

\section{Cambios en los contenidos didácticos}

Los avances tecnológicos presentan la información en una nueva configuración, permitiendo interactividad, combinando el texto escrito con sonido e imágenes y permitiendo la participación sinérgica de docentes y estudiantes como co-constructores de autores reconocidos al crear sus propios materiales de estudio (Laborda, 2005), como se advierte en la Figura 5. Otras fuentes de información pueden incluir bases de datos, repositorios unificados, bibliotecas digitales, repositorios de objetos de aprendizaje y laboratorios virtuales, entre otros.

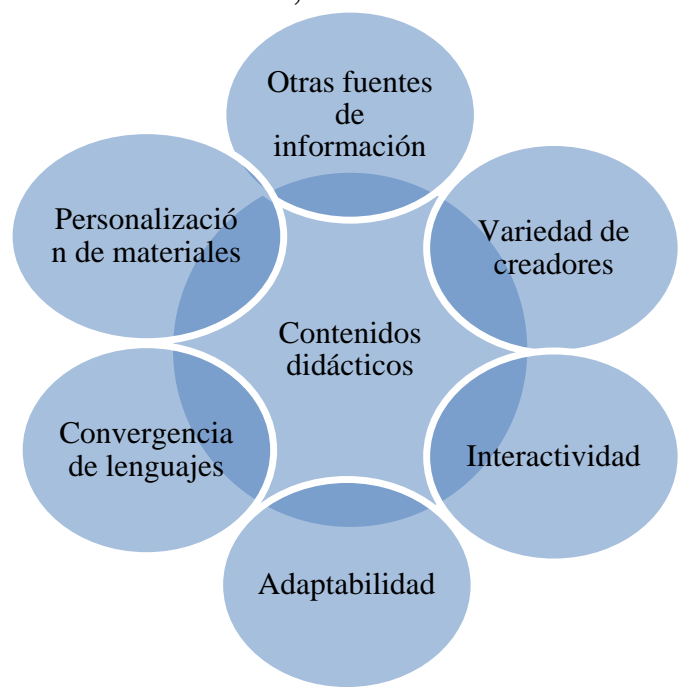

Figura 5, Factores que influyen en los contenidos didácticos, de Laborda (2005)

\section{El Conectivismo de George Siemens}

Para Siemens (2004), el conectivismo es la integración de principios explorados por las teorías de caos, redes, complejidad y auto-organización. El aprendizaje es un proceso que ocurre al interior de ambientes difusos de elementos centrales cambiantes - que no están por completo bajo control del individuo. El aprendizaje (definido como conocimiento aplicable) puede residir fuera de nosotros (al interior de una organización o una base de datos), está enfocado en conectar conjuntos de información especializada y las conexiones que nos permiten aprender más tienen mayor importancia que nuestro estado actual de conocimiento. 
El conectivismo es orientado por las decisiones basadas en principios que cambian rápidamente. Continuamente se está adquiriendo nueva información. La habilidad de distinguir entre la información importante y no importante resulta vital. También es crítica la habilidad de reconocer cuándo una nueva información altera un entorno basado en las decisiones tomadas anteriormente.

Principios del conectivismo:

1. El aprendizaje y el conocimiento dependen de la diversidad de opiniones.

2. El aprendizaje es un proceso de conectar nodos o fuentes de información especializados.

3. El aprendizaje puede residir en dispositivos no humanos.

4. La capacidad de saber más es más crítica que aquello que se sabe en un momento dado.

5. La alimentación y mantenimiento de las conexiones es necesaria para facilitar el aprendizaje continuo.

6. La habilidad de ver conexiones entre áreas, ideas y conceptos es una habilidad clave.

7. La actualización (conocimiento preciso y actual) es la intención de todas las actividades conectivistas de aprendizaje.

8. La toma de decisiones es, en sí misma, un proceso de aprendizaje. El acto de escoger qué aprender y el significado de la información que se recibe son vistos a través del lente de una realidad cambiante. Una decisión correcta hoy, puede estar equivocada mañana debido a alteraciones en el entorno informativo que afecta la decisión Siemens (2004).

El conocimiento puede ser descrito de muchas maneras. Pero la cuestión que más preocupa aquí es "dónde reside”. Siemens (2006: 11) señala: el conocimiento "permanece" en el individuo pero "reside” en el colectivo.

\section{Los Entornos Personales de Aprendizaje (PLE)}

Los Entornos Personales de Aprendizaje (PLE) constituyen una idea pedagógica sobre cómo aprenden las personas con tecnología. “...es el conjunto de herramientas, fuentes de información, conexiones y actividades que cada persona utiliza de forma asidua para aprender" (Adell y Castañeda, 2010, pág. 23).

El PLE no es tanto una tecnología como un enfoque sobre cómo emplear la tecnología actual para enseñar y para aprender. Como destaca Graham Attwell (citado en Castañeda y Adell, 2013) el creciente interés por los PLE, y otros usos de la tecnología en educación, podría deberse al desajuste entre lo que la sociedad demanda a las instituciones educativas y lo que éstas realmente entregan. A juicio de Castañeda y Adell (2013), se requiere un cambio radical de paradigma, desde una educación diseñada 
para satisfacer las necesidades de una sociedad industrial a una educación pensada en valores para una sociedad de la información y el conocimiento.

\section{Objetivos del estudio}

Los objetivos del presente proyecto se describen a continuación:

Analizar las propuestas del conectivismo que dan respuesta a los desafíos de la formación por competencias en el desarrollo de sistemas informáticos.

Identificar los resultados de la aplicación de los principios del conectivismo como metodología de enseñanza-aprendizaje.

\section{Metodología}

Para el estudio, de tipo cuali-cuantitativo, se propusieron las siguientes etapas metodológicas:

1. Selección del conjunto de competencias en las que se enfoca la intervención.

2. Selección de la asignatura que atienda al desarrollo de las competencias seleccionadas en el paso anterior y de los temas relevantes involucrados en ese proceso.

3. Selección de estrategias de enseñanza y evaluación apropiadas al desarrollo de las competencias seleccionadas y las herramientas TIC adecuadas para dichas estrategias.

4. Implementación de las estrategias y aplicación de las herramientas TIC conforme al diseño propuesto.

5. Evaluación de los resultados. Los resultados fueron evaluados en términos del grado de desarrollo de las competencias profesionales involucradas. Las evidencias que sustentaron la evaluación se resumen en: (a) la argumentación presentada en cada situación de decisión del aprendizaje auto-determinado, (b) el producto materializado en los trabajos prácticos, (c) los procedimientos aplicados para resolver cada una de las consignas (la indagación se realizó mediante la puesta en común grupal y las indicaciones consignadas en el blog), (d) las evidencias que respaldaron el desarrollo de la responsabilidad y (e) las evidencias que respaldaron el desarrollo de la solidaridad manifestada en las respuestas obtenidas a preguntas precisas formuladas por la cátedra.

A los fines prácticos, el proceso fue evaluado mediante la verificación de los siguientes criterios pensados en pro de la formación de los estudiantes y materializados en una lista de cotejo que contempló: (a) la manifestación de una actitud adecuada frente a la demanda académica, (b) la concreción de ideas y proyectos, (c) el diseño y desarrollo de software en el marco de los paradigmas actuales de programación en función de una demanda específica, (d) la transferencia de su proceso de aprendizaje a la 
vida cotidiana y (e) la colaboración con los miembros del equipo de trabajo y con la comunidad académica.

Por su parte, y a modo de complementar el estudio desde una perspectiva más integral, se procedió a adaptar y administrar un cuestionario elaborado por Cabero y Marín (2014), con el objetivo de conocer las percepciones que los alumnos tenían acerca del trabajo en equipo colaborativo. Los reactivos de los 31 ítems del instrumento contemplaron una escala comprendida entre "Totalmente en desacuerdo" hasta "Totalmente de acuerdo", pasando por “En desacuerdo", “Neutral” y "De acuerdo”. Cabe señalar que el estudio se complementó con las observaciones de los investigadores, cuya interpretación atendió al enfoque cualitativo del paradigma mixto aplicado.

De esta forma se arribó a una aproximación del rol del conectivismo en el aprendizaje auto-determinado, como estrategias de enseñanza y evaluación que refuerza la formación de competencias profesionales, considerando el desarrollo holístico del individuo.

\section{Descripción de los procedimientos}

La asignatura seleccionada fue Programación V, dado su afinidad con el desarrollo de las competencias adoptadas para el estudio y descritas a continuación:

1. Desarrolla software en el marco de los paradigmas actuales de programación en función de las demandas del mercado y el contexto organizativo.

2. Diseña algoritmos para la solución de problemas, aplicando los conceptos de los paradigmas de programación.

3. Sirve a sus semejantes y se prepara para el liderazgo al identificar las necesidades del curso y trabaja junto a los compañeros para encontrar soluciones, con una actitud altruista y solidaria, en el marco de la responsabilidad social y el compromiso ciudadano.

4. Formula y gestiona proyectos con una actitud emprendedora constituyéndose en un agente de transformación de su entorno.

El tamaño de la muestra se vio limitada por la cantidad de estudiantes que cursaron la asignatura durante el 2015, conformando, en su totalidad, un curso de 11 estudiantes. Dado el tamaño de la muestra, solo se pretende obtener un acercamiento a las respuestas buscadas, sin procurar su generalización.

En el desarrollo de la asignatura se aplicó el enfoque de aprendizaje heutagógico, el cual se centra en los estudiantes y sus experiencias previas de conocimiento obtenidos en asignaturas anteriores. Se basa en encontrar, descubrir y crear contenidos a fin de guiar un ejercicio investigativo. Esta forma de aprender pone énfasis en el aprender a aprender, utilizando 
procesos no lineales y la autodeterminación del aprendiz. A medida que el estudiante aprende, diseña y desarrolla el mapa del aprendizaje, incluye todos los nodos que aportan al acrecentamiento de sus conocimientos. A tal efecto utiliza distintas herramientas, mecanismos y actividades que le permiten obtener la información necesaria para el logro de los objetivos, tales como lo mencionan Adell y Castañeda (2010) al referirse a los PLE. Todo el proceso es acompañado por la reflexión que refuerza el aprendizaje autónomo y conduce a la obtención del producto que debe presentar como evidencia de la adquisición de las competencias en cuestión (aplicación software y portafolios), de la manera más eficientemente posible. Finalmente se compartieron los conocimientos obtenidos a través de: (a) una exposición en clase, en la que se explicó detalladamente tanto el proceso como el resultado y (b) un blog con el objetivo de llegar a estudiantes de otros ambientes académicos. En esta metodología los docentes se convierten en facilitadores del proceso de aprendizaje, creando ambientes propicios para la reflexión a partir de la formulación de preguntas esenciales y abiertas que promueven nuevos interrogantes.

Los criterios se resumen en (a) Argumentación, (b) Trabajos prácticos, (c) Procedimientos, (d) Responsabilidad y (e) Solidaridad.

\section{Resultados descriptivos}

Luego de obtenidos y tabulados los datos recabados, se inició la etapa de procesamiento de los mismos. La Tabla 1 refiere los estadísticos descriptivos de los cinco criterios seleccionados para el estudio. Las mayores medias se observan en Trabajos prácticos y Responsabilidad, mientras que en Solidaridad se advierte la media de menor valor.

Tabla 1: Estadísticos descriptivos

\begin{tabular}{cccc}
\hline & Media & Desviación típica & $\mathrm{N}$ \\
\hline Argumentación & 6,3627 &, 99916 & 11 \\
Trabajos Prácticos & 8,8455 &, 53919 & 11 \\
Procedimientos & 6,3636 & 1,12006 & 11 \\
Responsabilidad & 8,5527 & 1,64808 & 11 \\
Solidaridad & 6,1955 &, 89698 & 11 \\
\hline
\end{tabular}

\section{Resultados inferenciales: análisis de correlación}

Una vez obtenidas las valoraciones por los medios descritos, se procedió a analizar las posibles correlaciones existentes entre los criterios considerados. Una relación lineal positiva entre dos variables, indica que los valores de las dos variables varían en forma similar. A los efectos de obtener las evidencias, se recurrió a los coeficientes de correlación, que permiten cuantificar el grado de relación lineal existente entre dos variables cuantitativas y valorar el grado de ajuste de la nube de puntos a una recta. 
En las pruebas ejecutadas se aplicó el Coeficiente de Pearson, que toma valores entre -1 y 1 , puesto que es el coeficiente más utilizado para estudiar el grado de relación lineal existente entre dos variables.

El análisis de correlación lineal, que tuvo el propósito de identificar posibles relaciones entre las variables, arrojó los valores observados en la Tabla 2: (a) el valor del coeficiente de correlación de Pearson y (b) el nivel crítico bilateral correspondiente.

El nivel crítico permite decidir sobre la hipótesis nula de independencia lineal. Rechazamos la hipótesis nula de independencia cuando el nivel crítico es menor que el nivel de significación establecido de 0,05, concluyendo que existe relación lineal estadísticamente significativa.

De esta forma, basando el análisis en los coeficientes de correlación y en los niveles críticos asociados de la Tabla 2, se afirma que existe correlación significativa entre las variables consideradas en el estudio, dado que los coeficientes oscilan entre 0.630 y 0.980 , mientras que los niveles críticos, asociados a los estadísticos, son todos menores que 0,05.

Tabla 2: Correlaciones $(\mathrm{n}=11)$

$$
\text { Trabajos }
$$

\begin{tabular}{|c|c|c|c|c|c|c|}
\hline \multicolumn{2}{|c|}{ Variables } & Responsabilidad & Solidaridad & $\begin{array}{l}\text { Trabajos } \\
\text { Prácticos }\end{array}$ & Argumentación & Procedimientos \\
\hline \multirow{2}{*}{\multicolumn{2}{|c|}{$\begin{array}{c}\text { Responsabilidad Correlación } \\
\text { de Pearson } \\
\text { Sig. } \\
\text { (bilateral) }\end{array}$}} & 1 & ,630(*) &, $797(* *)$ &, $940(* *)$ &, $830(* *)$ \\
\hline & & & 038 & ,003 & ,000 & ,002 \\
\hline \multirow[t]{2}{*}{ Solidaridad } & $\begin{array}{l}\text { Correlación } \\
\text { de Pearson }\end{array}$ & ,630(*) & 1 & ,755(**) & ,674(*) & ,766(**) \\
\hline & $\begin{array}{l}\text { Sig. } \\
\text { (bilateral) }\end{array}$ & 038 & & 007 & ,023 & ,006 \\
\hline \multirow[t]{2}{*}{$\begin{array}{l}\text { Trabajos } \\
\text { Prácticos }\end{array}$} & $\begin{array}{l}\text { Correlación } \\
\text { de Pearson }\end{array}$ &, $797(* *)$ &, $755(* *)$ & 1 &, $866(* *)$ &, $980(* *)$ \\
\hline & $\begin{array}{l}\text { Sig. } \\
\text { (bilateral) }\end{array}$ & ,003 & 007 & & 001 &, 000 \\
\hline \multirow[t]{2}{*}{ Argumentación } & $\begin{array}{l}\text { Correlación } \\
\text { de Pearson }\end{array}$ &, $940(* *)$ & ,674(*) & ,866(**) & 1 &, $851(* *)$ \\
\hline & $\begin{array}{c}\text { Sig. } \\
\text { (bilateral) }\end{array}$ & 000 & ,023 & 001 & & ,001 \\
\hline \multirow[t]{2}{*}{ Procedimientos } & $\begin{array}{l}\text { Correlación } \\
\text { de Pearson }\end{array}$ &, $830(* *)$ & ,766(**) &, $980(* *)$ &, $851(* *)$ & 1 \\
\hline & $\begin{array}{c}\text { Sig. } \\
\text { (bilateral) }\end{array}$ & ,002 & ,006 & ,000 & ,001 & \\
\hline
\end{tabular}

Los datos recabados, acompañados de las pruebas estadísticas con sus correspondientes resultados, dan fe del desarrollo simultáneo de los atributos estudiados. Es decir que, para la muestra estudiada, se tiene evidencia del 
incremento de cada una de las variables consideradas, en la medida en que se observa el aumento de las restantes.

\section{Análisis de regresión}

A partir de la información obtenida del análisis de correlación, se procedió a aplicar el análisis de regresión lineal múltiple, como técnica estadística que permite estudiar la relación entre una variable dependiente y más de una variable independiente, con el doble propósito de (a) averiguar en qué medida la variable dependiente puede estar explicada por las variables independientes y (b) obtener predicciones de la variable dependiente a partir de las variables independientes.

A los efectos de analizar todas las combinaciones posibles, se procedió a trabajar cada una de las variables como variable dependiente y el resto como variables independientes, es decir que se reiteró la prueba para cada una de las variables incluidas en el estudio.

De las combinaciones posibles, se describen los resultados de aquellas pruebas cuyos resultados observaron el coeficiente de determinación cercano a uno y el estadístico $F$ asociado a un nivel crítico menor que 0,05, lo que permite identificar la existencia de una relación lineal significativa.

En primer lugar, se tomó la Responsabilidad como variable dependiente mientras que la Solidaridad, los Procedimientos, la Argumentación y los Trabajos prácticos, participaron como variables independientes. Se encontró que estas últimas explican un 90\% de la varianza de la Responsabilidad, pues, la $R^{2}$ corregida $=0,90$. El nivel crítico asociado al estadístico $F_{(4)}=23,48$ es de 0,001, indicando la existencia de una relación lineal significativa y, por lo tanto, un buen ajuste del hiper-plano, definido por la ecuación de regresión, a la nube de puntos definidos por los datos.

Los coeficientes Beta estandarizados indican la cantidad de cambio, en puntuaciones típicas, que se produce en la variable dependiente por cada cambio de unidad en la correspondiente variable independiente $\mathrm{y}$ manteniendo constantes el resto de las variables independientes. Estos coeficientes proporcionan información sobre la importancia relativa de cada variable independiente en la ecuación de regresión. En este caso, los coeficientes Beta indican que la variable Procedimientos es la más importante (1,204), seguida por Trabajos prácticos $(1,202)$ y por Argumentación (0,991). Por su parte los niveles críticos menores que 0,05 de las pruebas $t$, indican que se debe rechazar la hipótesis nula de que un coeficiente de regresión vale cero en la población. En los niveles críticos asociados a cada prueba $t$, se advierte que solo la variable Argumentación posee un coeficiente significativamente distinto de cero $(p=0,003)$. Los valores obtenidos dan evidencia de que solo la variable Argumentación es 
relevante en la ecuación de regresión y, por lo tanto, contribuye de forma significativa a explicar lo que ocurre con la variable Responsabilidad.

En segunda instancia, se tomó Procedimientos como variable dependiente mientras que la Solidaridad, la Responsabilidad, la Argumentación y los Trabajos prácticos, participaron como variables independientes, encontrándose que estas últimas explican un 97\% de la varianza de los Procedimientos, puesto que $R^{2}$ corregida $=0,967$. El nivel crítico asociado al estadístico $F_{(4)}=74,06$ es de 0,000 , indicando un buen ajuste del hiper-plano, definido por la ecuación de regresión, a la nube de puntos.

Los coeficientes Beta estandarizados indican que la variable Trabajos prácticos es la más importante $(0,962)$, seguida por Responsabilidad $(0,398)$ y por Argumentación (0,392). Por su parte en las observaciones de los niveles críticos asociados a cada prueba $t$, se advierte que solo la variable Trabajos prácticos posee un coeficiente significativamente distinto de cero $(p=0,000)$, por lo que se tiene evidencia de que solo esta variable es relevante en la ecuación de regresión y, por lo tanto, contribuye de forma significativa a explicar lo que ocurre con la variable Procedimientos.

En la tercera prueba se tomó Argumentación como variable dependiente mientras que Solidaridad, Responsabilidad, Procedimientos y Trabajos prácticos, participaron como variables independientes, encontrándose que estas últimas explican un 92\% de la varianza de la Argumentación, con una $R^{2}$ corregida $=0,918$. El nivel crítico asociado al estadístico $F_{(4)}=29,078$ es de 0,000 , indicando un buen ajuste del hiper-plano definido a la nube de puntos. Los coeficientes Beta estandarizados muestran que la variable Trabajos prácticos es la más importante $(1,131)$, seguida por Procedimientos $(0,967)$ y Responsabilidad (0,810). Los niveles críticos asociados a cada prueba $t$, revelan que solo las variables Responsabilidad $(p=0,003) \quad$ y Trabajos prácticos $(p=0,049)$ poseen coeficientes significativamente distintos de cero, por lo que se tiene evidencia de que estas únicas variables contribuyen de forma significativa a explicar lo que ocurre con la variable Argumentación.

En último lugar, se seleccionó Trabajos prácticos como variable dependiente mientras que Solidaridad, Responsabilidad, Procedimientos y Argumentación, participaron como variables independientes, encontrándose que estas últimas explican un 97\% de la varianza de los Trabajos prácticos, con una $R^{2}$ corregida $=0,968$. El nivel crítico asociado al estadístico $F_{(4)}=76,246$ es de 0,000 , indicando un buen ajuste a la nube de puntos. Los coeficientes Beta estandarizados muestran que la variable Procedimientos es la más importante (0,935), seguida por Argumentación $(0,445)$ y Responsabilidad (0,386). Los niveles críticos asociados a cada prueba $t$, revelan que solo las variables Procedimientos $(p=0,000)$ y Argumentación 
$(p=0,049)$ contribuyen de forma significativa a explicar lo que ocurre con la variable Trabajos prácticos.

A modo de descubrir el rol de la solidaridad en el proceso de aprendizaje auto-determinado, se analizó el comportamiento de los ítems adaptados del cuestionario de Cabero y Marín (2014), considerando para ello algunos de los ítems representativos de este atributo y la relación existente entre las potenciales variables en juego. De las combinaciones posibles, se encontró que los ítems "Me gusta que los cursos se desarrollen a través del trabajo en equipo" ( $p=0,02)$ y "Me gusta ayudar a otras personas al trabajar en equipo” ( $p=0,034)$, contribuyen de forma significativa a explicar lo que ocurre con la variable Responsabilidad, cuya varianza es explicada en un $60 \%$ por estos ítems.

Los resultados obtenidos muestran la interrelación entre gran parte de las variables estudiadas. Sin embargo, dada la limitación del tamaño de la muestra, los resultados deben ser interpretados con cautela, sin desatender las tendencias manifestadas por los datos recogidos.

Desde la interpretación de los resultados obtenidos, con la debida circunscripción a la muestra dado su tamaño limitado, se procedió a analizar la acción conjunta, sobre la variable Responsabilidad, de las variables (a) Argumentación, (b) Me gusta que los cursos se desarrollen a través del trabajo en equipo y (c) Me gusta ayudar a otras personas al trabajar en equipo; estas últimas como variables representantes de las preferencias por desarrollar los trabajos en equipo. Se encontró que las variables consideradas explican un 93\% de la varianza de Responsabilidad, con una $R^{2}$ corregida $=$ 0,929. El nivel crítico asociado al estadístico $F_{(3)}=44,387$ es de 0,000, indicando un buen ajuste a la nube de puntos. Los coeficientes Beta estandarizados muestran que la variable Argumentación es la más importante $(0,861)$, seguida por "Me gusta ayudar a otras personas al trabajar en equipo" $(0,270)$. Los niveles críticos asociados a cada prueba $t$, revelan que solo las variables Argumentación $(p=0,000)$ y Me gusta ayudar a otras personas al trabajar en equipo $(p=0,024)$ contribuyen significativamente a explicar lo que ocurre con la Responsabilidad. Es decir que estas últimas variables podrían mejorar la predicción de los valores de Responsabilidad en un 93\%.

\section{Discusión y conclusiones}

Las múltiples fuentes utilizadas para recolectar los datos y los métodos aplicados para convertirlos en información, permiten señalar algunos aspectos interesantes que a su vez podrían ser profundizados en estudios con muestras representativas a fin de rescatar posibles generalizaciones y convertirlas en recomendaciones convenientes para ser consideradas en el currículo académico. 
Se ha observado que la argumentación constituye una variable con posible poder predictivo sobre la responsabilidad de un estudiante. Este resultado conduce a suponer que cuando una argumentación tiene sustento sólido, da cuenta del cumplimiento previo de los requisitos de indagación de los contenidos abordados. Esto implicaría un proceso continuo de realimentación, donde el esfuerzo intencional proporciona los recursos que conducen al éxito de un emprendimiento.

Por su parte, cuando el estudiante adquiere habilidades para desarrollar con eficiencia los trabajos prácticos asignados, crea el andamiaje para construir procedimientos autónomos que robustecen la formación de las competencias esperadas; procesos reversibles que refuerzan el círculo virtuoso de responsabilidad y argumentación.

Si bien la solidaridad no apareció como variable predictiva de los atributos examinados, los análisis de correlación sugieren una estrecha vinculación entre ellos. Las observaciones de los investigadores permitieron advertir que aquellos estudiantes que mostraban predisposición al diálogo, al debate, al intercambio de ideas y al consenso, se esforzaban en mayor grado por participar en foros de discusión y, consecuentemente, obtenían información más relevante y actualizada que aquellos en quienes no se observó esta cualidad. La apertura hacia el aprendizaje colaborativo facilitó el desarrollo de las competencias esperadas y propició el fortalecimiento de las relaciones interpersonales. La construcción de estas redes, con la correcta elección de nodos, redundó en resultados que confirman que el aprendizaje consiste en crear una red donde los nodos se conectan para dar forma a una compleja fuente de conocimiento. La medida de dicha complejidad permite contemplar de un modo más real, y acabado, la realidad en la que se espera actuar.

Se estima que la metodología de aprendizaje auto-determinado, acompañado por los recursos que proporciona el conectivismo, crea un escenario ventajoso como fundamento de la formación profesional que persigue el desarrollo de las competencias que la sociedad demanda de las instituciones educativas.

Las correlaciones estadísticamente significativas entre las variables en juego, sugieren el desarrollo simultáneo de los atributos representados. Estos hallazgos dan evidencia, para esta muestra, que las cualidades estudiadas se van nutriendo entre ellas y que no resultan de la improvisación sino del esmero y esfuerzo que cada individuo invierte no solo en su propia formación sino también en la formación de los demás compañeros que componen su red de aprendizaje.

Por lo expuesto, puede advertirse las bondades del conectivismo, y sus recursos, integradas a una metodología de aprendizaje auto-determinado 
donde se presentan las condiciones óptimas para desarrollar una red de interacciones en el marco de la formación holística del ser.

\section{References:}

Adell, J. y Castañeda, L. (2010). “Los Entornos Personales de Aprendizaje (PLEs): una nueva manera de entender el aprendizaje”. En Roig Vila, R. y Fiorucci, M. (Eds.) Claves para la investigación en innovación y calidad educativas. La integración de las Tecnologías de la Información y la Comunicación y la Interculturalidad en las aulas. Alcoy: Marfil - Roma TRE Universita degli studi.

Barragán Sánchez, R., Mimbrero Mallado, C. y Pacheco González-Piñal, R. (julio, 2013). Cambios pedagógicos y sociales en el uso de las IC: u-learning y u-portafolio. Revista Electrónica de Investigación y Docencia (REID), 10, 2013, 7-20.

Bartolomé, A. (2011). Conectivismo: aprender em rede e na rede. En Marcelo Brito Carneiro Leão: Tecnologias na Educaçao: Uma abordagem crítica para uma atuaçao práctica. Recife (Brasil): UFRPE, 71-86.

Cabero, Julio y Marín, Verónica (2014). Posibilidades educativas de las redes sociales y el trabajo en grupo: Percepciones de los alumnos universitarios, Revista Científica de Educomunicación, 42, 165-172.

Castañeda, L. y Adell, J. (Eds.). (2013). Entornos Personales de Aprendizaje: claves para el ecosistema educativo en red. Alcoy: Marfil.

Laborda, M. R. (2005). Las Nuevas Tecnologías en la Educación. Cuadernos/sociedad de la Educación 5. Fundación AUNA. Madrid, España.

Marquès Graells, P. (2006). Criterios de calidad para los sistemas de teleformación. Plantilla de evaluación. Obtenido de Internet el 28 de marzo de 2012 de: http://www.peremarques.net/stfcalid.htm

Padrón, José y Ortega, Alfredo. (2012). La conectividad: Dogmatismo o nuevo referente paradigmático para el docente de vanguardia. Revista de Investigación, 75(36), 129-142.

Siemens, G. (2004). Conectivismo: Una teoría de aprendizaje para la era digital. Obtenido de: http://www.diegoleal.org/docs/2007/Siemens(2004)Conectivismo.doc

Siemens, G. (2006). Conociendo el conocimiento. Obtenido de: http://craig.com.ar/ biblioteca/Conociendo\%20el\%20Conocimiento\%20\%20George\%20Siemens.pdf 